Bio-grafia: Escritos sobre la Biologia y su Enseñanza Vol 4 No. 6 ISSN 2027-1034. Primer semestre de 2011, Bogotá, Colombia, pp 110 -124.

\title{
PERTINENCIA DE ELABORAR COLECCIONES BIOLÓGICAS: UNA ESTRATEGIA QUE FORTALECE UNA ACTITUD INVESTIGATIVA HACIA LA CONSERVACIÓN BIOLÓGICA
}

\section{RELEVANCE OF PREPARE BIOLOGICAL COLLECTIONS A STRATEGY THAT STRENGTHENS A RESEARCHABLE ATTITUDE TOWARDS BIOLOGICAL CONSERVATION}

Por: Gloria Esperanza Díaz Medina

\section{Resumen}

\begin{tabular}{|l|}
\hline Recibido: $26-03-2011$ \\
\hline Aceptado: 07-07-2011 \\
\hline
\end{tabular}

Este trabajo es resultado de la práctica pedagógica y presenta un análisis sobre la pertinencia que estudiantes de Licenciatura en Biología le dan a la elaboración de una colección biológica, para lo cual, se aplicaron ocho guías de laboratorio relacionadas con la diversidad animal y vegetal, tomando aspectos teóricos sobre biodiversidad y conservación con el objeto de fomentar una actitud investigativa hacia la conservación biológica. Además, se aplicaron dos escalas Likert, centradas en cuatro categorías de análisis (conceptual, procedimental, valor educativo, valor intrínseco). Finalmente, se obtuvo que para fomentar una actitud investigativa hacia la conservación es necesario orientar procesos de cuestionamiento, curiosidad, observación y reflexión al momento de ver la pertinencia de las colecciones biológicas al abordar temas sobre biodiversidad

Palabras clave: Actitud Investigativa, Biodiversidad, Colección Biológica, Conservación Biológica, Escala Likert, Valor Educativo, Valor Intrínseco.

\begin{abstract}
This paper is the result of the pedagogical practicum and presents an analysis about the relevance that undergraduate biology students give to the development of a biological collection, for which eight laboratory guidelines related to animal and plant diversity were implemented, taking theoretical aspects of biodiversity and conservation with the aim of fostering a research attitude towards biological conservation. In addition to, two Likert scales were applied, which were focused on four categories of analysis (conceptual, procedural, educational value, intrinsic value). Finally, it was found that to
\end{abstract}

'Estudiante de Licenciatura en Biología. X semestre. Universidad Pedagógica Nacional. Bogotá. Colombia. Correo: gloesdime@yahoo.es. 
encourage a researchable attitude towards conservation it is necessary to guide processes of questioning, curiosity, observation and reflection when the relevance of biological collections in approaching biodiversity issues is tackled.

Key words: Researchable Attitude, Biodiversity, Biological Collection, Biological Conservation, Likert Scale, Educational Value, Intrinsic Value.

\section{INTRODUCCIÓN.}

La práctica pedagógica como espacio de formación se caracteriza por diversas relaciones que se establecen entre el profesor y estudiante. Pero desde la experiencia en este ambiente ¿Qué profesor no desearía que sus estudiantes mostraran un alto interés por su asignatura? Pues bien, la bibliografía señala que estudiantes lo van perdiendo el interés a medida que pasa el tiempo (Osborne et.al, 1998 citado en Gavidia, 2008), es decir, ese interés por aprender alguna asignatura va disminuyendo con el tiempo, por lo cual es pertinente conocer los factores que generan esto y así buscar alternativas que motive en los estudiantes aprender. Es así como, el presente trabajo enfatiza lo que se observa a nivel universitario, como por ejemplo, se evidencia una fortaleza al momento de enseñar, para el caso de tercer semestre de licenciatura en Biología, y es que un conocimiento para que adquiera la importancia y comprensión necesaria, debe ser pertinente dentro del contexto de la población con la cual se esté trabajando.

En consecuencia, se aborda el tema de colección biológica no sólo por el poco conocimiento que se tiene sobre las colecciones del departamento de biología desde lo analizado en tercer semestre, sino también, porque dentro del contexto que se maneja en este semestre es pertinente, dado a que, le proporciona al estudiante asociar lo teórico con lo práctico a nivel de la diversidad biológica. Con la anterior premisa, en calidad de profesora, se propuso partir también si para los estudiantes es pertinente o no elaborar colecciones biológicas, lo cual fue analizado relacionando la investigación, biodiversidad y conservación desde 4 aspectos principalmente: conceptual, procedimental, valor educativo y valor intrínseco.

Se eligió como estrategia metodológica la elaboración de colecciones, dado a que tiene gran impacto en lo educativo al momento de realizar y analizar una problemática. Además, porque en tercer semestre, se orienta un trabajo "hacia la formación de un ciudadano que valore la naturaleza estérica e intelectual, y que al reconocer el valor de la diversidad adopte una actitud respetuosa... y se haga participe de la construcción social de identidad en lo político, lo social y lo cultural" (Jessup. et.al, 1999), y por ello, se busca contribuir al desarrollo de estos aspectos dado al compromiso que se tiene como futuros educadores. Asimismo, a pesar que al momento de elaborar colecciones biológicas se busca "preservar para futuras generaciones (...), realizar un inventario completo de la biodiversidad; comprender la diversidad biológica, crear bases de datos para el manejo de la información (...) y creación de redes informáticas a través de las cuales fluya la información en 
todas partes" (Crisci, 2006), la intención con los estudiantes fue analizar las implicaciones que tiene pensar en elaborar una colección, con el fin de integrar a su formación en qué momentos debe ser o no pertinente, pero siempre partiendo desde lo que piensan y construyen en cada laboratorio; todo esto converge en un sólo objetivo común "el uso sustentable de y la conservación de la biodiversidad" como menciona Crisci (2006).

Por lo anterior, algunos de los conceptos involucrados con las colecciones biológicas son los temas de biodiversidad y conservación biológica. Así que, se entiende por biodiversidad la variedad y variabilidad de seres vivos y ecosistemas que éstos integran, los cuales están organizados en tres niveles: genes, especies y ecosistemas (Crisci, 2006). Pero desde un punto de vista social, se toma la biodiversidad como un componente "intangible", es decir, todo conocimiento, práctica e innovación individual o colectiva, asociado a dicha diversidad (Suarez, 1997). En efecto, el concepto de biodiversidad resalta la estrecha relación entre diversidad biológica y diversidad cultural y reconoce la importancia de las actividades humanas para la producción y conservación de los recursos biológicos, los cuales son de suma importancia al hablar en un contexto educativo.

Para el caso de la conservación biológica se tiene en cuenta que está dentro de un campo de acción que es la biología de la conservación, precisamente debido a, que se nutre de varias disciplinas para desarrollar métodos interdisciplinarios prácticos encaminados a la protección, preservación, y recuperación de la biodiversidad (Primack, 2002), aún sin tener como tal un fundamento teórico como campo de conocimiento. Por ello, se toma a la biología de la conservación como "una síntesis de las disciplinas científicas que se ocupan de la actual crisis de la biodiversidad. Combina enfoques de investigación básica y aplicada para impedir la pérdida de la biodiversidad (p.76). Intenta dar respuesta a preguntas específicas aplicables a situaciones reales (p. 22)" (Primack, 2002).

Ahora bien, algunas de las razones por las que es valioso hacer una colección biológica, es que éstas han contribuido con las causas de la conservación de la biodiversidad de muchas formas. Por ejemplo, los especímenes en una colección pueden ser reanalizados cuantas veces sea necesario, ayudando a conservar la biodiversidad al no tener que obtener un ilimitado número del mismo tipo de espécimen, al mismo tiempo que se minimizan los costos de operación y una mayor eficiencia en los trabajos de campo (Páez, 2004). Además, han sido beneficiosas, tanto para el mundo natural como para la sociedad, desempeñando un papel vital en aspectos de salud humana (estudio de patógenos, vectores de enfermedades), monitoreo de cambios ambientales (seguimiento a contaminantes ambientales y cambio climático global), y por supuesto en el avance de las ciencias biológicas, incluyendo estudios de la biodiversidad y su pérdida (Páez, 2004). De igual manera, representan el patrimonio natural de un país o región, constituyen un archivo histórico natural de utilidad múltiple donde la preservación de especímenes y su información asociada son la base de estudios taxonómicos, sistemáticos, ecológicos, filogenéticos, biogeográficos, de genética de poblaciones y conservación; forman parte 
fundamental en el conocimiento de la diversidad biológica y en el avance de las ciencias biológicas (Tobar, 2002 citado en Mesa, s.f).

Además de integrar las colecciones biológicas en el estudio de la diversidad, se busca fomentar una actitud investigativa en los estudiantes, con el objeto que empiecen a profundizar sus conocimientos analizando cada situación de manera reflexiva, Por ello se entiende por actitud investigativa como la predisposición cog-noscitiva, emocional y social que se asume consciente y permanentemente frente a todos los elementos conceptuales, metodológicos y técnicos propios del quehacer investigativo (Cerda, 2010), lo cual, contribuye a orientar los laboratorios y formas de analizar dos pre- y post-test, a manera de escala Likert que se aplicaron durante la práctica, los cuales tuvieron el propósito de reconocer actitudes investigativas en torno a la conservación biológica e importancia o no de las colecciones biológicas. $\mathrm{Y}$ posteriormente corroborar el satisfactorio desarrollo de una actitud investigativa que relacione la pertinencia que tiene elaborar una colección biológica en el contexto educativo.

El presente documento propone fomentar una actitud investigativa hacia la conservación biológica en estudiantes de tercer semestre de licenciatura en Biología, analizando la pertinencia o no de elaborar una colección biológica. Esta propuesta parte de los contenidos del semestre durante los espacios de laboratorio, para con ello fortalecer lo planteado en el eje curricular de tercer semestre, que es "reconocer parte de la diversidad local y cómo algunas comunidades la usan, la valoran y la integran a sus tradiciones y creencias; reconocer que como parte de la diversidad biológica, los humanos compartimos los mismos problemas y necesidades de las otras especies y finalmente para que al conocer la diversidad local, se tengan más elementos al momento que las comunidades deban tomar decisiones en torno a su conservación"(García y Ladino, 2009 ).

\section{MATERIALES Y MÉTODOS}

Durante la práctica pedagógica se trabajó con 48 estudiantes de tercer semestre de Licenciatura en Biología, de los cuales sólo a 24 estudiantes se les aplicó dos escalas Likert y ocho guías de laboratorio, estas guías fueron orientadas a partir de la estrategia “ciclo de indagación" según Enseñanza de la Ecología en el Patio de la Escuela (EEPE), la cual emplea una "pedagogía constructivista de <aprender haciendo> donde cada estudiante construye su propio conocimiento a través de la acción y el contenido temático se adquiere en el proceso" (Feinsinger. et.al, 2002). Es pertinente esta estrategia en la medida que contribuye a fomentar actitudes investigativas hacia la conservación y analizar la pertinencia o no de elaborar una colección biológica.

El presente trabajo fue de carácter cuantitativo con alguna inclusión de datos cualitativos (Lerma, 2004) para su análisis. El enfoque cuantitativo, se aplicó mediante dos escalas Likert a manera de pre-test y post-test, los cuales fueron analizados estadísticamente, dando cuenta si hubo o no fomento de actitudes investigativas hacia la conservación 
biológica y pertinencia o no de hacer una colección biológica por parte de los estudiantes. El enfoque cualitativo, se orientó a partir de observación participante y ocho guías de laboratorio, las guías se realizaron según las tres etapas del ciclo de indagación (planteamiento de la pregunta problema, acción y reflexión). Sin embargo, sólo se les da la información de cada tema y la pregunta problema a través de las guías de laboratorio, luego ellos construyen la información que permita responder a la pregunta, durante el tiempo de laboratorio el cual fue teórico-práctico; y finalmente para que llevaran a la reflexión todo el trabajo realizado, se evaluó mediante una pregunta en cada laboratorio como fututo educador.

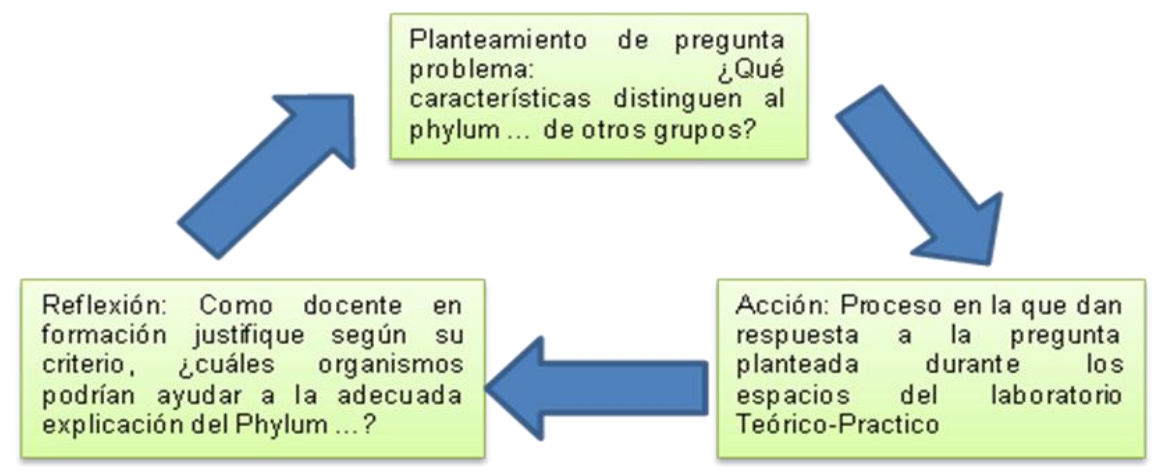

\section{Gráfica 1. Esquema general aplicado en las guías de laboratorio}

Además, para determinar en el contexto del semestre la pertinencia que los estudiantes le dan a elaborar colecciones biológicas, dentro del análisis cuantitativo y cualitativo se tuvo en cuenta relacionar la investigación, biodiversidad y conservación desde 4 aspectos principalmente: a. Conceptual. Se centró en mirar cómo están entendiendo la colección biológica, la conservación biológica y la biodiversidad; b. Procedimental. Se buscó ver si ellos le dan pertinencia o no en hacer una colección biológica y en qué medida, al momento de aplicar los laboratorios; c. Valor educativo. Según lo actitudinal se pretendió evidenciar si para ellos elaborar colecciones biológicas contribuye al estudio de temas relacionados con la ciencia y otras áreas de conocimiento diferentes a la Biología; d. Valor intrínseco. Entendiéndolo como "valor no instrumental", o valor como fin en sí (Arribas, 2006), es decir, según lo actitudinal se pretendió evidenciar si los estudiantes le otorgan valor a las demás especies solo por el hecho de existir, al correlacionar la pertinencia o no de elaborar una colección biológica.

\section{RESULTADOS Y DISUSIÓN}

En el anexo $\mathrm{N}^{\circ} 1$ se presenta el formato de la primera escala Likert cuyo objeto fue reconocer tanto los conocimientos como las actitudes que muestran los estudiantes frente a la biodiversidad, conservación biológica, colección biológica e investigación. Para ello se hace énfasis en su valor educativo e intrínseco. 
Ahora bien, la primer escala Likert, muestra que gran parte de los estudiantes logran reconocer que describir especies del planeta es un objetivo de la conservación biológica (Figura $\mathrm{N}^{\circ} 3$, ítem 5) y según Crisci (2006), éste es un aspecto de importancia en relación con la conservación de la biodiversidad, al mencionar que para evitar la extinción de una especie, el primer paso es conocerla científicamente, es decir, describirla. Frente a esto los estudiantes muestran un cambio favorable en su actitud hacia investigar, de acuerdo con la afirmación: "Si observo en campo una especie que está en extinción la colecto para hacer una colección biológica" (Figura $\mathrm{N}^{\circ} 2$, Ítem 11) el cual está representado con un alto porcentaje a diferencia del pre-test aplicado, dado que reflexionan sobre el previo conocimiento que se debe tener acerca de los organismos a colectar y su lugar de ubicación.
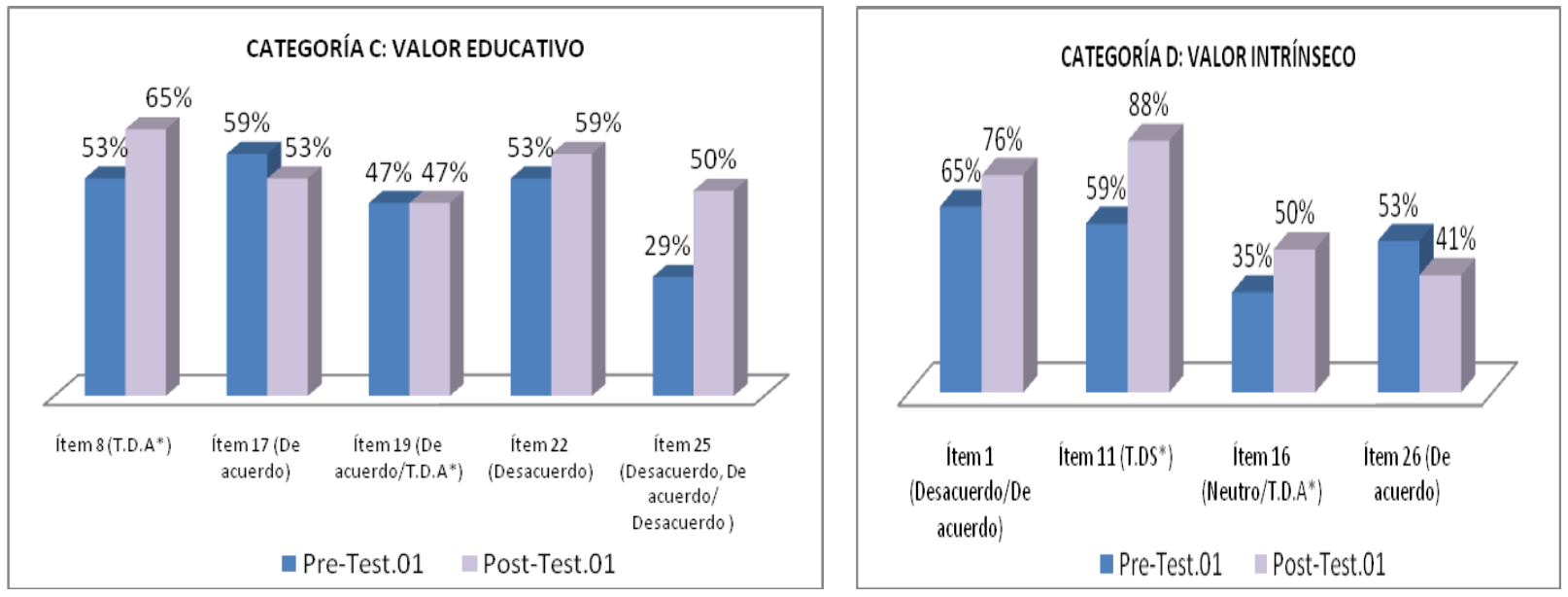

Figura $N^{\circ} 1$ y 2. T.D.A*, Totalmente De Acuerdo.T.DS*, Totalmente en desacuerdo.

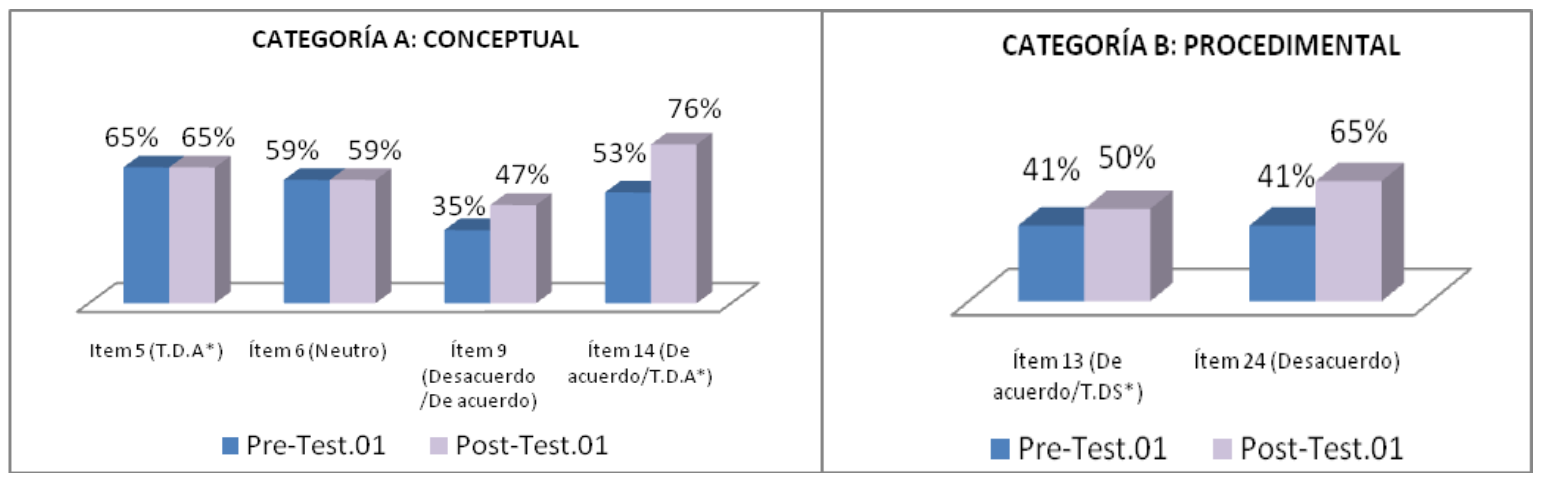

Figura N³ y 4 T.D.A*, Totalmente De Acuerdo. T.DS*, Totalmente en desacuerdo.

En contraste con lo anterior, los estudiantes resaltan la pertinencia de hacer colecciones biológicas a nivel educativo haciendo distinción entre algunos grupos como por ejemplo a nivel de microorganismos mencionan que "un cultivo, como ejemplo de pertinencia en una colección biológica es fundamental, puesto que al implementarlo como método de enseñanza nos 
permite llevar un seguimiento más detallado de un proceso tan importante como lo es el desarrollo de un ser vivo en su totalidad, en este caso de colonias " (González. et.al. 2010). Estos aspectos dan cuenta de la forma en que están dando valor a las colecciones y su pertinencia, entonces vemos allí reflejado el valor educativo e intrínseco; en donde el cambio de actitud desde el valor intrínseco se evidencia al entender que solo la existencia de algún organismo es muy importante y entender su desarrollo aporta bastante conocimiento, lo cual no es visto como un valor instrumental desde la utilidad para un beneficio económico sino desde el valor educativo.

Por ello, vemos que los fines e intereses humanos son muy diversos, así como los medios para satisfacerlos. Por ejemplo, no es lo mismo talar un bosque por completo para enriquecerse rápidamente que entresacar los mejores árboles mientras se plantan otros nuevos. Tampoco se mide igual el valor instrumental que otorgamos al bosque como "almacén" de madera que el valor instrumental que le atribuimos como proveedor de paz espiritual, goce estético o sublimación mística. Y, por supuesto, el valor instrumental que se le atribuye como proveedor de un hábitat adecuado para ciertas especies animales, como ecosistema que absorbe gases nocivos o que evita la erosión del suelo, etc. Como vemos, hay una gran variedad de concepciones instrumentales del valor y gran parte de ellas son inconmensurables entre sí: no admiten una gradación cardinal, aunque de continuo nos veremos impelidos a establecer una ordenación jerárquica por las exigencias de satisfacción de nuestras necesidades y deseos. (Arribas, 2006)

En este orden de ideas, los estudiantes ven un aporte de las colecciones biológicas desde el valor educativo a nivel investigativo, en la medida que afirman que "las colecciones biológicas son pertinentes, puesto que contribuyen de una manera activa y dinámica a los procesos académicos, al lograr que la teoría no quede en los imaginarios, sino que se contraste con especímenes reales llevando a un plano tangible lo teórico (...) donde a nivel investigativo contribuye a complementar los inventarios de la biodiversidad del planeta" (Buelvas. et.al. 2010). Esto nos muestra que el valor instrumental aquí está mediado no en lo económico sino en lo investigativo y en actitud para lograr que el conocimiento y las formas de aprenderlo y enseñarlo haga parte de nuestro quehacer.

En el anexo $\mathrm{N}^{\circ} 2$, se presenta el formato de la segunda escala Likert, aplicado a estudiantes del Grupo 01. Componente Diversidad. Tercer semestre. Licenciatura en biología. Universidad Pedagógica Nacional, cuyo objetivo fue determinar las actitudes que los estudiantes tienen en relación con la elaboración de micro preparados y montajes de microorganismos, donde se evidenció si hay pertinencia o no de tener en cuenta este enfoque dentro de las colecciones biológicas. Para ello se hace énfasis en su valor educativo e intrínseco los cuales se relacionan posteriormente con las categorías conceptual y procedimental. 


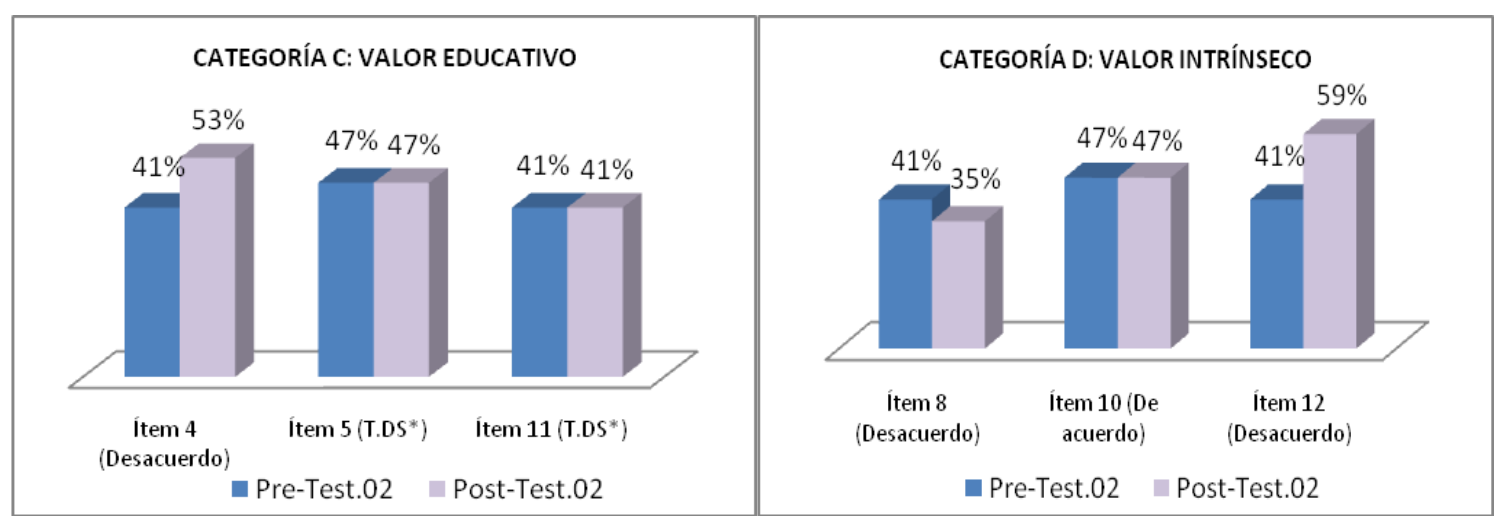

Figura N5 Y 6. T.DS*, Totalmente en desacuerdo.

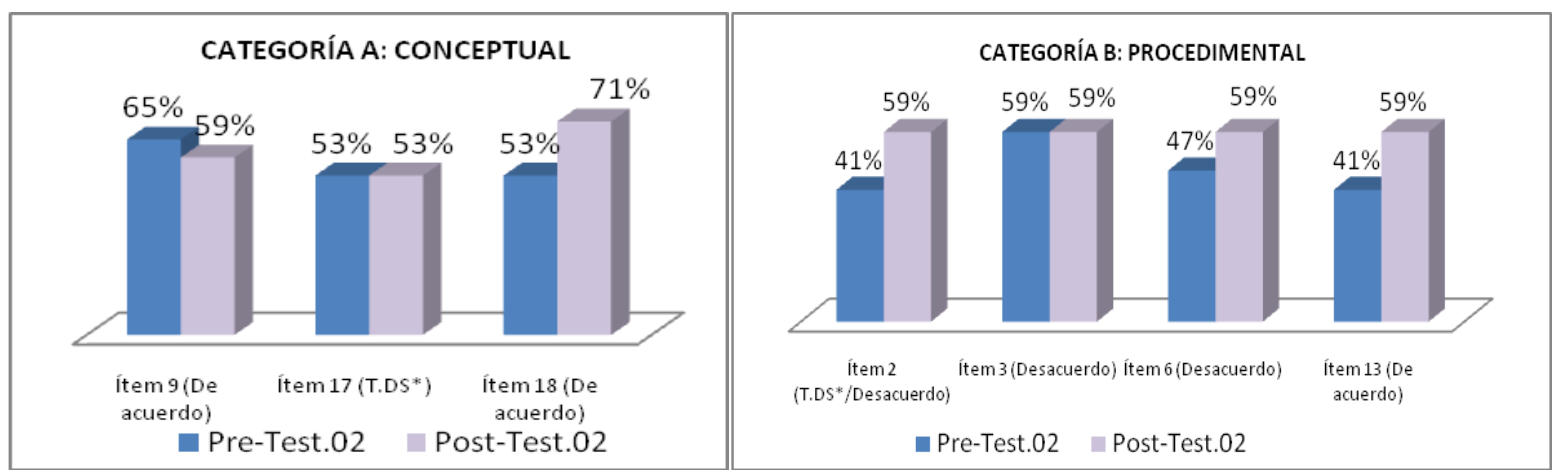

\section{Figura $N^{\circ} 7$ y 8 . T.DS*, Totalmente en desacuerdo.}

Por lo anterior, las 4 categorías de análisis (conceptual, procedimental, valor educativo, valor intrínseco) aplicadas para las dos escalas Likert, permitieron evidenciar que el fomento de actitudes investigativas hacia la conservación debe partir de comprender lo conceptual, para luego construir desde dónde se puede ver la pertinencia o no de hacer una colección biológica. Así pues, no se reduce lo actitudinal a lo conceptual sino que se destacan otras dimensiones que de alguna $u$ otra manera son parte fundamental en la formación de futuros docentes en el ambiente de la biodiversidad.

En consecuencia, vemos que las actitudes son necesarias para cualquier aprendizaje; se mantienen y refuerzan por la valoración positiva del mismo. De ahí que la actitud puede considerarse causa y efecto del aprendizaje. No obstante, entender las actitudes exclusivamente ligadas a determinados contenidos sería equipararlas a la motivación y por lo tanto reducir sus dimensiones. Las actitudes poseen otros objetivos : la contribución al desarrollo personal del individuo mediante la adquisición de valores, que son principios de norma, guía de conducta ante situaciones que implican elección, las predisposiciones estables y positivas de la personalidad. (Gavidia, 2008) 
Visto que el valor intrínseco no es el valor utilitario sino todo lo contrario, los estudiantes reflejan en la escala Likert $\mathrm{N}^{\circ} 2$ (Figura $\mathrm{N}^{\circ}$ 6, Ítem 8), que hubo un considerable cambio en este tipo de actitud, con respecto al pre-test, dado a que en el post-test hay un mayor porcentaje en Desacuerdo, y Totalmente en desacuerdo; con respecto a la afirmación: "Sólo es necesario conocimiento biológico para ver pertinencia de elaborar colecciones", ya que dan cuenta que no sólo desde la biología sino desde otras disciplinas se puede abordar la pertinencia de las colecciones biológicas y si lo vemos desde la conservación es totalmente aceptable y relevante tener claridad de este aspecto, puesto que la biología de la conservación no tiene un fundamento teórico sino que aplica medidas y juicios de valor con el aporte de diferentes disciplinas. Cabe señalar, a nivel procedimental por ejemplo; en la escala Likert $\mathrm{N}^{\circ} 2$ (Figura $\mathrm{N}^{\circ} 8$, Ítem 3), que los estudiantes mostraron un considerable cambio de actitud, debido a que en el post-test hay mayor porcentaje en Desacuerdo, y Totalmente en desacuerdo, frente a la afirmación: "Basta con elaborar un micro preparado para entender mejor la teoría", reflejando que aunque es importante la elaboración de micro preparados no es lo único pertinente para entender un tema.

Finalmente, a partir de lo que se asume como actitud investigativa; se observó cómo es ese proceso investigativo por parte del estudiante al momento de indagar un tema y hacer un laboratorio, dado que, confronta su saber cotidiano con el conocimiento disciplinar para luego construir la pertinencia o no de algunos organismos, con el objeto de hacer una colección biológica y que diera cuenta de su utilidad (no productiva). Esto se evidenció por las 2 escalas Likert aplicadas (categorías B, C, y D) como en las guías de laboratorio. En relación a las guías de laboratorio los estudiantes mencionan que "las implicaciones de hacer una colección biológica de artrópodos podría incidir bastante a la hora de explicar la conservación de los mismos; hay muchos organismos pertenecientes a este Phylum que se encuentran amenazados o en peligro, por lo que sería preferible remitirse a una colección biológica ya estructurada, que permitiera identificar las características del Phylum sin alterar la permanencia de estas especies" (Saavedra. et.al, 2010). Lo anterior nos muestra que antes de elaborar una colección se debe tener conocimiento de lo que se busca colectar, dado que hay organismos que se encuentran en peligro y si se colecta sin hacer una revisión previa si ya lo tienen en una colección pues el papel de la colección biológica quedaría en el coleccionismo que es un aspecto totalmente diferente a lo que se presente en la academia con las colecciones biológicas. La afirmación que hace Saavedra y otros, explica que si es importante por parte de los estudiantes ver primero la en qué medida es pertinente elaborar colecciones biológicas frente a un tema especifico desde lo que se pueda investigar sobre el grupo a colectar, para luego mirar las posibilidades de hacer una colección desde una mirada de la conservación.

\section{CONCLUSIONES}


Se logró evidenciar con los laboratorios que los estudiantes dan gran significado al aprendizaje de los temas, pensando desde la pertinencia de elaborar una colección biológica, debido a que los hace reflexionar como futuros educadores en la medida que muestran aportes desde la conservación y lo educativo como por ejemplo; mencionar que los ejemplares que se tomarían para abordar una temática y elaborar una colección serían "aquellos cuyas características que identifica al grupo sean altamente visibles para evidenciar las diferencias entre este grupo y otro" (Ariza. et.al, 2010). Por tanto, para fomentar una actitud investigativa hacia la conservación es necesario orientar procesos de cuestionamiento, curiosidad, observación y reflexión al momento de ver la pertinencia de las colecciones biológicas al abordar temas sobre biodiversidad

De hecho, los laboratorios mostraron que la elaboración de colecciones biológicas por parte de los estudiantes al tener un carácter de desarrollo individual y grupal, permite apropiar de una manera más dinámica y experiencial la importancia y pertinencia de las colecciones y la conservación biológica, donde a través del fomento de una actitud investigativa y junto con la observación en campo, laboratorio y desde lo cotidiano, es posible dar sentido y valor al conocimiento de la biodiversidad y su conservación, debido a que, el componente diversidad "busca fortalecer el conocimiento de un grupo local e incrementar las colecciones del museo y herbario" (García y Ladino, 2009), pero aquí la intención fue dar relevancia al proceso que tiene la preparación de las colecciones biológicas.

\section{AGRADECIMIENTOS}

Agradezco la culminación del presente trabajo de práctica a mi titular Martha García por sus esfuerzos y acompañamiento constante durante los laboratorios y colaboración con el desarrollo del las actividades en el componente Diversidad. Eje curricular Diversidad. Licenciatura en Biología. Universidad Pedagógica Nacional, por sus aportes y orientación en torno a las colecciones biológicas. A Sonia Martínez asesora del énfasis faunística y conservación de artrópodos, por sus constantes esfuerzos en orientar mi proceso pedagógico e interpretación de lo actitudinal en relación a las colecciones biológicas. También a la Profesora Nubia Ladino que dirige la parte de vegetal en el componente, por su interés, conocimiento y colaboración con el desarrollo de los laboratorios y finalmente a los estudiantes del grupo 01 del componente (periodo II-2009; periodo I-2010), por sus aportes cuestionamientos y discusiones hechas durante los laboratorios y en general todas las clases, pues sin ellos no se hubiera podido visualizar desde qué parámetros es pertinente la elaboración de colecciones biológicas y su valor educativo.

\section{REFERENCIAS BIBLIOGRÁFICAS}

Arribas, F. (2006). Del Valor Intrínseco de la Naturaleza. Isegoría, Enero-Junio, 34, 261-275. 
Ariza, L., González, C., Hoyos, C., Pulido, E., Peña, N., Sánchez P. (2010). Informe de laboratorio: Protozoa. Periodo I. Grupo 01. Tercer semestre. Licenciatura en Biología. Universidad Pedagógica Nacional.

Buelvas, I., García, L., Gutiérrez, L., Ramírez, G. (2010). Informe de laboratorio: Cnidaria. Periodo I. Grupo 01. Tercer semestre. Licenciatura en Biología. Universidad Pedagógica Nacional.

Cerda, H. (2010). Un camino hacia la formación integral e innovativa en la investigación científica. En. Red internacional de actitud y cultura investigativa. Disponible en: http://rediaci.org/

Crisci, J. (2006). Espejos de Nuestra Época: Biodiversidad, Sistemática y Educación. En Rev. Gayana Bot. 63. N 1. Págs. 106-114.

Feinsinger, P., Arango, N., Chávez M. (2002). Guía metodológica para la enseñanza de ecología en el patio de la escuela. Nueva York: Audubon.

García, M., Ladino, N. (2009). Programa: Componente Diversidad I. Tercer semestre. Periodo II. Departamento de Biología. Facultad de Ciencia y Tecnología. Universidad Pedagógica Nacional. Bogotá.

Gavidia, V. (2008). Las actitudes en la educación científica. Didáctica de las Ciencias Experimentales y Sociales, 22, 53-66.

González, C., Ariza, L., Hoyos, C., Pulido, E., Peña, N., Sánchez P. (2010). Informe de laboratorio: Cnidaria. Periodo I. Grupo 01. Tercer semestre. Licenciatura en Biología. Universidad Pedagógica Nacional.

Jessup, M. et.al. (1999). El proyecto curricular de la licenciatura. Licenciatura en Biología. Proyecto Curricular. Departamento de Biología. Facultad de Ciencia y Tecnología. Universidad Pedagógica Nacional. Bogotá. p 82.

Lerma H. (2004). Metodología de la investigación: propuesta, anteproyecto y proyecto (3 $3^{\mathrm{a}}$ ed.). Bogotá: Ecoe.

Mesa, D. (2005). Protocolos para la preservación y manejo de colecciones biológicas. Boletín Científico - Centro de Museos - Museo de Historia Natural. Enero- Diciembre 1 (10). Disponible en: http://boletincientifico.ucaldas.edu.co/downloads/Revista\%2010_6.pdf 
Páez V. (2004). El valor de las Colecciones Biológicas. En Rev: Actualidades Biológicas. Universidad de Antioquia. Volumen 26 (81) / julio - diciembre. Recuperado el 10 Octubre 2009 de http://matematicas.udea.edu.co/ actubiol/resumenes/26 81.html

Primack, R. (2002). Introducción a la Biología de la Conservación. España: Editorial Ariel., p $21,22,76$.

Saavedra, B., González, H., Pulido, E., Cifuentes, C., Quispe, I. (2010). Informe de laboratorio: Arthropoda. Periodo I. Grupo 01. Tercer semestre. Licenciatura en Biología. Universidad Pedagógica Nacional.

Suárez, L. (1997). La importancia de la biodiversidad. Biodiversidad, Bioprospección y Bioseguridad. Quito-Ecuador: ABYA-YALA, p 17-22.

\title{
ANEXO N 1 ESCALA LIKERT 1: ACTIVIDAD PRE Y POST-TEST № 1-4 LA DIVERSIDAD DE LO VIVO EN LAS COLECCIONES BIOLÓGICAS Estudiantes Grupo 01. Componente Diversidad. III semestre Licenciatura en biología Universidad Pedagógica Nacional
}

\author{
Nombre: $\quad$ código:
}

Cuando se afecta un ecosistema o desaparece una planta o un animal, se rompen los frágiles hilos de la telaraña de la vida, tejida a lo largo de miles de millones de años de evolución. Por consiguiente, es importante observar en detalle que la conservación es un tema ligado a la biodiversidad, donde tener en cuenta aspectos de colección biológica inciden e igual manera en el aprendizaje de estos temas.

Lea con atención cada una de las siguientes afirmaciones. Marque de 1 a 5, teniendo en cuenta su experiencia:

1: Totalmente en desacuerdo

2: En desacuerdo

3: Sin opinión (se declara neutro)
4: 4: De acuerdo

5: 5: Totalmente de acuerdo

\begin{tabular}{|r|l|l|l|l|}
\cline { 3 - 4 } \multicolumn{2}{l|}{} & \multicolumn{1}{l|}{\begin{tabular}{l}
1 \\
\multicolumn{2}{l}{}
\end{tabular}} & 2 & 4 \\
1 & $\begin{array}{l}\text { Aprender sobre biodiversidad es lo más importante para la } \\
\text { elaboración de una colección biológica. }\end{array}$ & & & \\
\hline 2 & $\begin{array}{l}\text { La elaboración de una colección biológica permite enseñar más } \\
\text { fácil sin abordar los conceptos a profundidad. }\end{array}$ & & & \\
\hline 3 & La investigación generalmente no contribuye a la protección de & & & \\
\hline
\end{tabular}


Bio-grafia: Escritos solore la Biologia y su Enseñanza Vol 4 No. 6 ISSN 2027-1034. Primer semestre de 2011, Bogotá, Colombia, pp 110 -124.

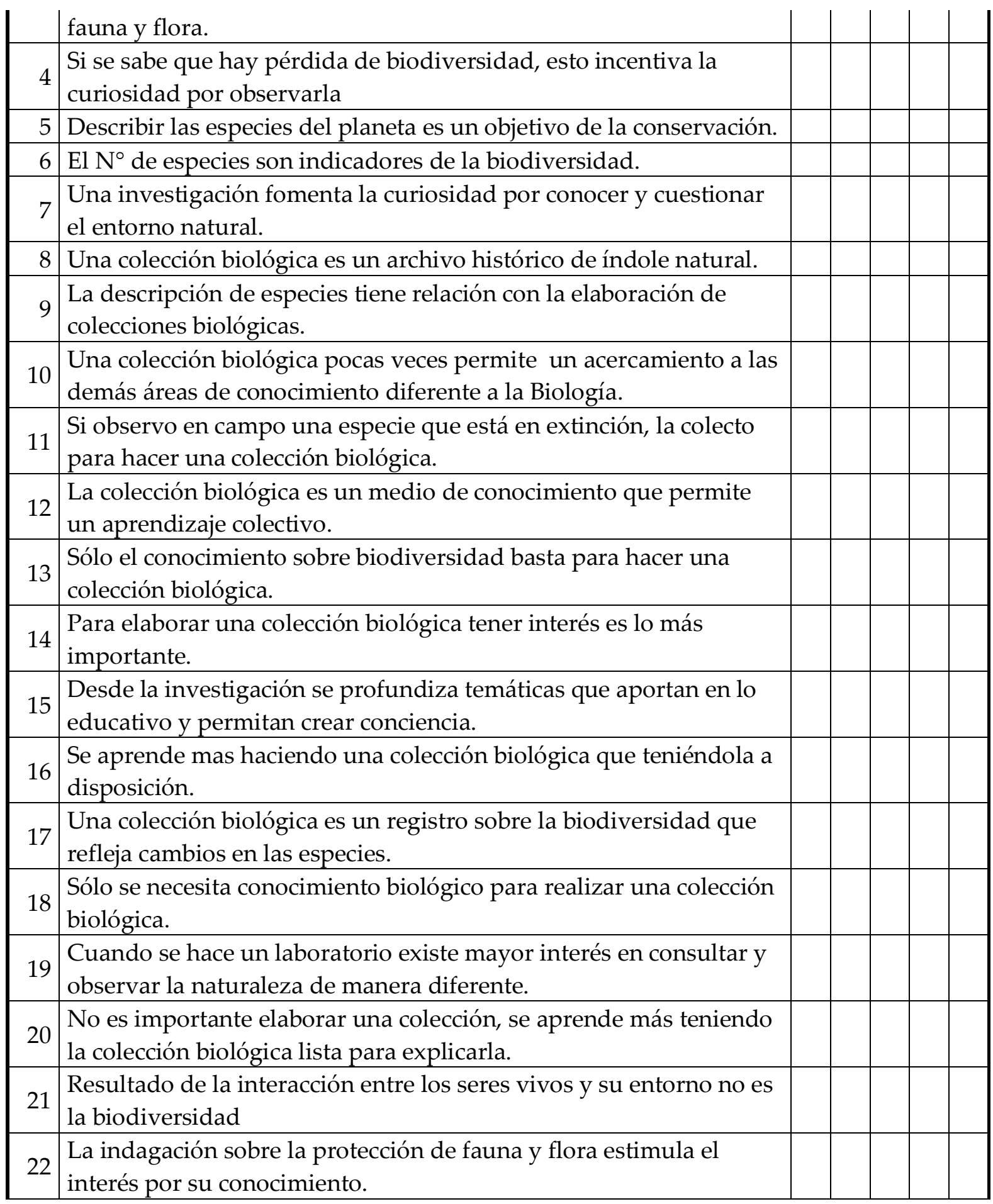


Bío-grafia: Escritos solve la Biologia y su Enseñanza Vol 4 No. 6 ISSN 2027-1034. Primer semestre de 2011, Bogotá, Colombia, pp 110 -124.

\section{ANEXO N ${ }^{\circ} 2$ \\ ESCALA LIKERT 2: ACTIVIDAD PRE Y POST-TEST № 2 \\ EL JUEGO DE LOS MICROPREPARADOS EN LA COLECCIÓN BIOLÓGICA \\ Estudiantes Grupo 01. Componente Diversidad. III semestre \\ Licenciatura en biología \\ Universidad Pedagógica Nacional}

Nombre:

Código:

Lea con atención cada una de las siguientes afirmaciones. Puntúe cada una de ellas con una escala de 1 a 5 , donde cada puntuación corresponde a lo cercano que una afirmación le describa a usted, teniendo en cuenta la más apropiada:

1: Totalmente en desacuerdo

2: En desacuerdo

3: Sin opinión (se declara neutro)
4: De acuerdo

5: Totalmente de acuerdo

\begin{tabular}{|c|c|c|c|c|c|c|}
\hline & & 1 & 2 & 3 & 4 & 5 \\
\hline 1 & Es colección biológica hacer micro preparados. & & & & & \\
\hline 2 & $\begin{array}{l}\text { Desarrollar técnicas de manejo en microscopía no contribuye a } \\
\text { interpretar la importancia de los montajes y micro preparados en el } \\
\text { aula. }\end{array}$ & & & & & \\
\hline 3 & $\begin{array}{l}\text { Basta con elaborar un micro preparado para entender mejor la } \\
\text { teoría. }\end{array}$ & & & & & \\
\hline 4 & $\begin{array}{l}\text { No es importante hacer montajes, se aprende más teniendo un } \\
\text { montaje en fresco o micro preparado listo para explicarlo. }\end{array}$ & & & & & \\
\hline 5 & Solo educativamente es importante elaborar micro preparados. & & & & & \\
\hline 6 & Solo basta tener micro preparados para desarrollar una clase. & & & & & \\
\hline 7 & Cuando se hace un micro preparado se piensa en conservar. & & & & & \\
\hline 8 & $\begin{array}{l}\text { Sólo es necesario conocimiento biológico para ver pertinencia de } \\
\text { elaborar colecciones. }\end{array}$ & & & & & \\
\hline 9 & $\begin{array}{l}\text { Elaborar micro preparados contribuye distinguir más elementos de } \\
\text { lo vivo. }\end{array}$ & & & & & \\
\hline 10 & $\begin{array}{l}\text { Hay más curiosidad por analizar lo vivo a nivel microscópico al } \\
\text { terminar de hacer un micro preparado en clase. }\end{array}$ & & & & & \\
\hline 11 & $\begin{array}{l}\text { Se aprende mejor la teoría que haciendo micro preparados como } \\
\text { colección biológica. }\end{array}$ & & & & & \\
\hline 12 & $\begin{array}{l}\text { Al momento de hacer montajes pocas veces permite un } \\
\text { acercamiento a la dinámica de lo vivo. }\end{array}$ & & & & & \\
\hline 13 & $\begin{array}{l}\text { La realización de montajes en fresco permite complementar lo } \\
\text { teórico. }\end{array}$ & & & & & \\
\hline
\end{tabular}


Bío-grafia: Escritos solve la Biologia y su Enseñanza Vol 4 No. 6 ISSN 2027-1034. Primer semestre de 2011, Bogotá, Colombia, pp 110 -124.

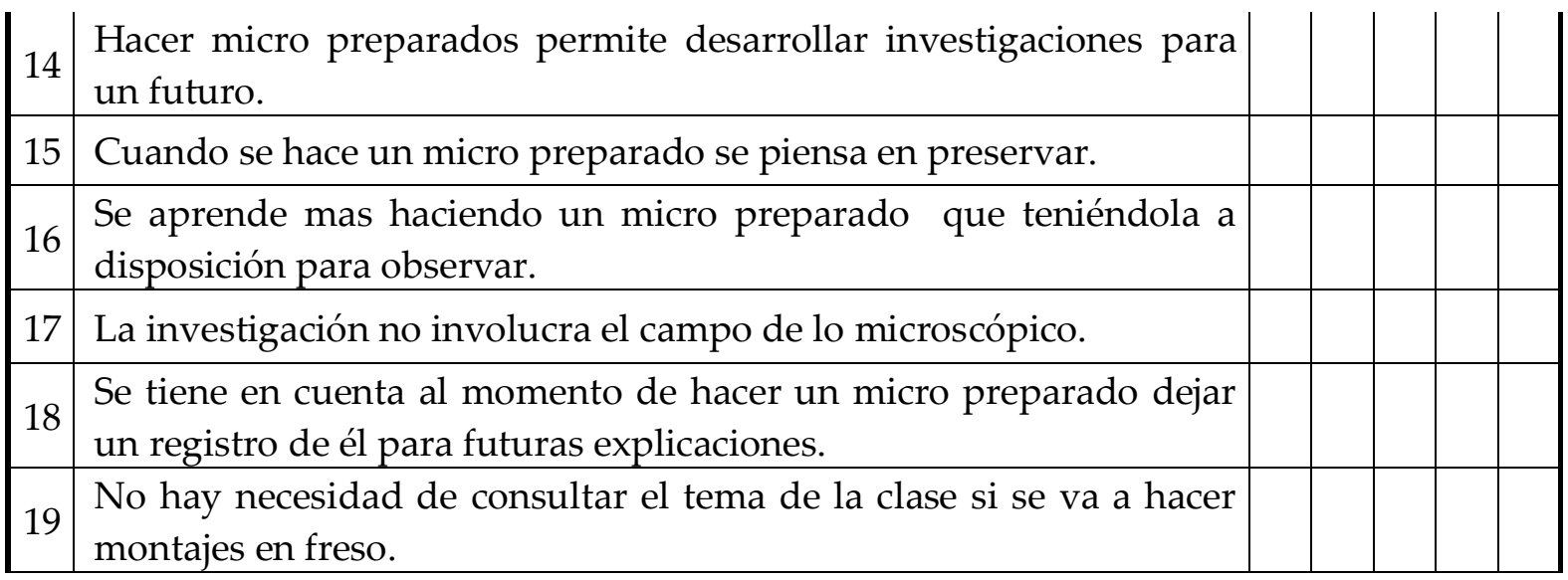

